\title{
Two-body charmed baryon decays involving decuplet baryon in the quark-diagram scheme
}

\author{
Y. K. Hsiao ${ }^{a}$, Qian Yi, Shu-Ting Cai, H. J. Zhao \\ School of Physics and Information Engineering, Shanxi Normal University, Linfen 041004, China
}

Received: 9 July 2020 / Accepted: 11 November 2020 / Published online: 19 November 2020

(C) The Author(s) 2020

\begin{abstract}
In the quark-diagram scheme, we study the charmed baryon decays of $\mathbf{B}_{c} \rightarrow \mathbf{B}^{*} M$, where $\mathbf{B}_{c}$ is $\Lambda_{c}^{+}$ or $\mathbf{\Xi}_{c}^{+(0)}$, together with $\mathbf{B}^{*}(M)$ the decuplet baryon (pseudoscalar meson). It is found that only two $W$-exchange processes are allowed to contribute to $\mathbf{B}_{c} \rightarrow \mathbf{B}^{*} M$. Particularly, we predict $\mathcal{B}\left(\Lambda_{c}^{+} \rightarrow \Sigma^{* 0(+)} \pi^{+(0)}\right)=(2.8 \pm$ $0.4) \times 10^{-3}$, which respects the isospin symmetry. Besides, we take into account the $S U$ (3) flavor symmetry breaking, in order to explain the observation of $\mathcal{B}\left(\Lambda_{c}^{+} \rightarrow \Sigma^{*+} \eta\right)$. For the decays involving $\Delta^{++}\left(\right.$иии), we predict $\mathcal{B}\left(\Lambda_{c}^{+} \rightarrow\right.$ $\left.\Delta^{++} \pi^{-}, \Xi_{c}^{+} \rightarrow \Delta^{++} K^{-}\right)=(7.0 \pm 1.4,13.5 \pm 2.7) \times 10^{-4}$ as the largest branching fractions in the singly Cabibbosuppressed $\Lambda_{c}^{+}, \boldsymbol{\Xi}_{c}^{+} \rightarrow \mathbf{B}^{*} M$ decay channels, respectively, which are accessible to the LHCb, BELLEII and BESIII experiments.
\end{abstract}

\section{Introduction}

To determine the mass and lifetime of the $\Lambda_{b}$ baryon, $\Lambda_{c}^{+}$ is often taken as the final state in the $\Lambda_{b}$ decays [1], which involves the favored Cabibbo-Kobayashi-Maskawa (CKM) matrix elements for bigger branching fractions. With the higher precision in the recent years [2,3], the subsequent $\Lambda_{c}^{+} \rightarrow p K^{-} \pi^{+}$decay has helped to make more accurate observations for the $\Lambda_{b}$ decays, which receives the significant contribution from $\Lambda_{c}^{+} \rightarrow \Delta^{++} K^{-}, \Delta^{++} \rightarrow p \pi^{+}$. Similarly, one uses $\Xi_{b}^{0} \rightarrow \Xi_{c}^{+} \pi^{-}$to determine the $\Xi_{b}^{0}$ lifetime, whereas we find that the subsequent process $\Xi_{c}^{+} \rightarrow p K^{-} \pi^{+}$ and its resonant contribution from $\Xi_{c}^{+} \rightarrow \Delta^{++} K^{-}, \Delta^{++} \rightarrow$ $p \pi^{+}$have not been well studied yet [1]. Therefore, $\mathbf{B}_{c} \rightarrow$ $\mathbf{B}^{*} M$ plays the key role in the precision measurements for the multi-body decays of beauty and charm baryons, where $\mathbf{B}_{c}=\left(\Lambda_{c}^{+}, \Xi_{c}^{+(0)}\right), \mathbf{B}^{*}$ the decouplet baryon and $M$ the meson state, such as $\Lambda_{c}^{+}\left(\Xi_{c}^{+}\right) \rightarrow \Delta^{++} K^{-}$.
The $\mathbf{B}_{c} \rightarrow \mathbf{B}^{*} M$ decays are not richly observed. Therefore, it is still unclear how the $\mathbf{B}_{c} \rightarrow \mathbf{B}^{*} M, \mathbf{B}^{*} \rightarrow \mathbf{B} M^{\prime}$ decays mix with the non-resonant contributions to $\mathbf{B}_{c} \rightarrow$ $\mathbf{B} M M^{\prime}$. In addition, $\mathbf{B}_{c} \rightarrow \mathbf{B} V, V \rightarrow M M^{\prime}$ with $V$ the vector meson causes more complicated mixtures [1]. The $S U$ (3) flavor $\left(S U(3)_{f}\right)$ symmetry has been widely applied to the charmed baryon decays [4-16]. By well explaining the data, the flavor symmetry does not appear to be severely broken in $\mathbf{B}_{c} \rightarrow \mathbf{B} M$ [17], where $\mathbf{B}$ denotes the octet baryon. By contrast, $\mathcal{B}\left(\Lambda_{c}^{+} \rightarrow \Sigma^{*+} \eta\right)$ not well interpreted by the $S U(3)_{f}$ symmetry might hint the broken effect in $\mathbf{B}_{c} \rightarrow \mathbf{B}^{*} M$ [7], which could be as large as that in the $D$ meson decays [1821].

For a better understanding of the hadronization in $\mathbf{B}_{c} \rightarrow$ $\mathbf{B}^{*} M$, there have been some theoretical attempts, which are in terms of the pole model, quark model and irreducible $S U$ (3) flavor $\left(S U(3)_{f}\right)$ symmetry [4-7, 22-24]. Particularly, the quark-diagram scheme with the topological $S U(3)_{f}$ symmetry provides a clear picture for the decay processes $[17,25-$ 27]. Due to the fact that $\mathbf{B}^{*}$ is a spin-3/2 baryon with totally symmetric quark contents, it can be shown that the topological diagrams involving the flavor anti-symmetric quark pair in $\mathbf{B}^{*}$ are forbidden or suppressed. Therefore, we purpose to use the quark-diagram scheme to relate all possible $\mathbf{B}_{c} \rightarrow$ $\mathbf{B}^{*} M$ decay channels. With the existing data, we will perform the numerical analysis, and determine different topological contributions. We can hence test the validity of the topological scheme, which involves the $S U(3)_{f}$ symmetry and its broken effect. Furthermore, we will give predictions for $\mathcal{B}\left(\mathbf{B}_{c} \rightarrow \mathbf{B}^{*} M\right)$ to be compared to the future measurements, which can help to clarify how $\mathbf{B}_{c} \rightarrow \mathbf{B}^{*} M, \mathbf{B}^{*} \rightarrow \mathbf{B} M^{\prime}$ mixes with $\mathbf{B}_{c} \rightarrow \mathbf{B} V, V \rightarrow M M^{\prime}$ and the non-resonant configuration in $\mathbf{B}_{c} \rightarrow \mathbf{B} M M^{\prime}$.

\footnotetext{
a e-mail: yukuohsiao@gmail.com (corresponding author)
} 


\section{Formalism}

\subsection{Effective Hamiltonian in the flavor symmetry}

To study the two-body charmed baryon decays, the corresponding quark-level effective Hamiltonian is given by [28]

$\mathcal{H}_{e f f}=\frac{G_{F}}{\sqrt{2}} \sum_{i=1,2} c_{i}\left(\lambda_{a} O_{i}^{a}+\lambda_{p} O_{i}^{p}+\lambda_{c} O_{i}^{c}\right)$,

with $\lambda_{(a, p, c)} \equiv\left(V_{c s}^{*} V_{u d}, V_{c p}^{*} V_{u p}, V_{c d}^{*} V_{u s}\right)$ and $p=(d, s)$, where $G_{F}$ is the Fermi constant, and $c_{i}$ the Wilson coefficients. The current-current operators $O_{i}^{(a, p, c)}$ are written as

$$
\begin{aligned}
& O_{1}^{a}=(\bar{u} d)(\bar{s} c), \quad O_{2}^{a}=\left(\bar{u}_{\beta} d_{\alpha}\right)\left(\bar{s}_{\alpha} c_{\beta}\right), \\
& O_{1}^{p}=(\bar{u} p)(\bar{p} c), \quad O_{2}^{p}=\left(\bar{u}_{\beta} p_{\alpha}\right)\left(\bar{p}_{\alpha} c_{\beta}\right), \\
& O_{1}^{c}=(\bar{u} s)(\bar{d} c), \quad O_{2}^{c}=\left(\bar{u}_{\beta} s_{\alpha}\right)\left(\bar{d}_{\alpha} c_{\beta}\right),
\end{aligned}
$$

where $\left(\bar{q}_{1} q_{2}\right)=\bar{q}_{1} \gamma_{\mu}\left(1-\gamma_{5}\right) q_{2}$, and the subscripts $(\alpha, \beta)$ denote the color indices. With $s_{c} \equiv \sin \theta_{c} \simeq 0.22$, where $\theta_{c}$ denotes the Cabibbo angle for the quark-mixing in the weak interaction, the decays with $\left|\lambda_{(a, p, c)}\right| \simeq\left(1, s_{c}, s_{c}^{2}\right)$ are regarded as the Cabibbo-allowed (CA), singly Cabibbosuppressed (SCS) and doubly Cabibbo-suppressed (DCS) processes, respectively.

For the lowest-lying anti-triplet charmed baryon states $\Xi_{c}^{0}$, $\Xi_{c}^{+}$and $\Lambda_{c}^{+}$that consist of $(d s-s d) c,(s u-u s) c$ and $(u d-$ $d u) c$, respectively, we present them as

$\mathbf{B}_{c}=\left(\begin{array}{ccc}0 & \Lambda_{c}^{+} & \Xi_{c}^{+} \\ -\Lambda_{c}^{+} & 0 & \Xi_{c}^{0} \\ -\Xi_{c}^{+} & -\Xi_{c}^{0} & 0\end{array}\right)$.

The pseudoscalar meson states are given by

$M=\left(\begin{array}{ccc}\frac{1}{\sqrt{2}}\left(\pi^{0}+c \phi \eta+s \phi \eta^{\prime}\right) & \pi^{-} & K^{-} \\ \pi^{+} & \frac{-1}{\sqrt{2}}\left(\pi^{0}-c \phi \eta-s \phi \eta^{\prime}\right) & \bar{K}^{0} \\ K^{+} & K^{0} & -s \phi \eta+c \phi \eta^{\prime}\end{array}\right)$,

where $\left(\eta, \eta^{\prime}\right)$ mix with $\eta_{q}=\sqrt{1 / 2}(u \bar{u}+d \bar{d})$ and $\eta_{s}=$ $s \bar{s}$. The mixing angle $\phi=(39.3 \pm 1.0)^{\circ}$ in $(s \phi, c \phi) \equiv$ $(\sin \phi, \cos \phi)$ comes from the mixing matrix, given by [29, 30]

$$
\left(\begin{array}{c}
\eta \\
\eta^{\prime}
\end{array}\right)=\left(\begin{array}{cc}
\cos \phi & -\sin \phi \\
\sin \phi & \cos \phi
\end{array}\right)\left(\begin{array}{l}
\eta_{q} \\
\eta_{s}
\end{array}\right)
$$

The decuplet baryons are written as
By neglecting the Lorentz indices, $\mathcal{H}_{\text {eff }}$ for the $c \rightarrow$ $q_{i} \bar{q}_{j} q_{k}$ transition can be presented with the tensor notation, $H_{j}^{k i}$, and the nonzero entries are given by [27]

$H_{2}^{31}=\lambda_{a}, \quad H_{2}^{21}=\lambda_{d}, \quad H_{3}^{31}=\lambda_{s}, \quad H_{3}^{21}=\lambda_{c}$.

\subsection{The quark-diagram scheme}

In the quark-diagram scheme, there exist six different topological diagrams for the $\mathbf{B}_{c} \rightarrow \mathbf{B}^{(*)} M$ decays, as drawn in Fig. 1a-f, parameterized as the topological amplitudes $\left(T, C, C^{\prime}\right)$ and $\left(E^{\prime}, E_{\mathbf{B}}, E_{M}\right)$, respectively [17]. More explicitly, $T$ and $C^{(\prime)}$ proceed with the $W$-boson emission ( $\left.W_{\mathrm{EM}}\right)$. By exchanging the $W$ boson $\left(W_{\mathrm{EX}}\right)$, it gives rise to $E^{\prime}$ and $E_{\mathbf{B}(M)}$. Since only $(T, C)$ can be decomposed of two separate matrix elements based on the factorization, that is, $(T, C) \propto\left\langle M\left|\bar{q}_{1 q_{2}}\right| 0\right\rangle\left\langle\mathbf{B}^{(*)}\left|\bar{q}_{3} c\right| \mathbf{B}_{c}\right\rangle$ [31], one classifies $(T, C)$ and $\left(C^{\prime}, E^{\prime}, E_{M, \mathbf{B}}\right)$ as the factorizable and nonfactorizable amplitudes, respectively.

Furthermore, it is found in Fig. 1a, b that $\mathbf{B}_{c}$ with $\left(q_{a} q_{b}-\right.$ $\left.q_{b} q_{a}\right) c$ cannot be turned into $\mathbf{B}^{*}\left(q_{a} q_{b} q_{k(i)}\right)$, where $q_{a} q_{b} q_{k(i)}$ are totally symmetric, such that $(T, C)$ give no contributions to $\mathbf{B}_{c} \rightarrow \mathbf{B}^{*} M$. Thus, the $\mathbf{B}_{c} \rightarrow \mathbf{B}^{*} M$ decays are purely non-factorizable processes. In addition, $C^{\prime}$ and $E^{\prime}$ are suppressed in $\mathbf{B}_{c} \rightarrow \mathbf{B}^{*} M$ [25], which is in accordance with the Körner-Pati-Woo theorem [32-34]. With the currentcurrent structure of $\left(\bar{q}_{i} q_{j}\right)_{V-A}\left(\bar{q}_{k} c\right)_{V-A}$ in Eq. (2), $q_{i}$ and $q_{k}$ are color anti-symmetric. When combined as the constituents of the baryon, $q_{i, k}$ are flavor anti-symmetric, such that the topological diagrams $\left(C^{\prime}, E^{\prime}\right)$ in Fig. 1c, d contribute to $\mathbf{B}_{c} \rightarrow \mathbf{B} M$, instead of $\mathbf{B}_{c} \rightarrow \mathbf{B}^{*} M$. Consequently, we are left with the $W_{\mathrm{EX}}$ topological diagrams $\left(E_{\mathbf{B}}, E_{M}\right)$ in Fig. 1e, $\mathrm{f}$ for $\mathbf{B}_{c} \rightarrow \mathbf{B}^{*} M$.

To proceed, we derive the amplitudes as $\mathcal{A}\left(\mathbf{B}_{c} \rightarrow\right.$ $\left.\mathbf{B}^{*} M\right)=\left(G_{F} / \sqrt{2}\right) T\left(\mathbf{B}_{c} \rightarrow \mathbf{B}^{*} M\right)$. Explicitly, the $T$ amplitudes ( $T$-amps) read $[17,25,27]$

$$
\begin{aligned}
T\left(\mathbf{B}_{c} \rightarrow \mathbf{B}^{*} M\right)= & E_{\mathbf{B}}^{(s)}\left(\mathbf{B}_{c}\right)^{j a} H_{j}^{k i}\left(\mathbf{B}^{*}\right)_{k a b}(M)_{i}^{b} \\
& +E_{M}^{(s)}\left(\mathbf{B}_{c}\right)^{j a} H_{j}^{k i}\left(\mathbf{B}^{*}\right)_{i a b}(M)_{k}^{b},
\end{aligned}
$$

where the parameters $E_{\mathbf{B}, M}^{(s)}$ correspond to the topological diagrams in Fig. 1e, f, respectively. The $W_{\mathrm{EX}}$ decay process needs an additional quark pair from $g \rightarrow q \bar{q}$, where $q \bar{q}$ could be $u \bar{u}, d \bar{d}$ or $s \bar{s}$. To take into account the broken $S U(3)_{f}$ symmetry, $E_{\mathbf{B}(M)}$ with $g \rightarrow s \bar{s}$ can be more specifically denoted by $E_{\mathbf{B}(M)}^{S}$. Under the exact $S U(3)_{f}$ symmetry, it leads to $E_{\mathbf{B}(M)}^{s}=E_{\mathbf{B}(M)}$ [26]. In Tables 1, 2 and 3, we

$$
\mathbf{B}^{*}=\frac{1}{\sqrt{3}}\left(\left(\begin{array}{ccc}
\sqrt{3} \Delta^{++} & \Delta^{+} & \Sigma^{*+} \\
\Delta^{+} & \Delta^{0} & \frac{\Sigma^{* 0}}{\sqrt{2}} \\
\Sigma^{*+} & \frac{\Sigma^{* 0}}{\sqrt{2}} & \Xi^{* 0}
\end{array}\right),\left(\begin{array}{ccc}
\Delta^{+} & \Delta^{0} & \frac{\Sigma^{* 0}}{\sqrt{2}} \\
\Delta^{0} & \sqrt{3} \Delta^{-} & \Sigma^{*-} \\
\frac{\Sigma^{* 0}}{\sqrt{2}} & \Sigma^{*-} & \Xi^{*-}
\end{array}\right),\left(\begin{array}{ccc}
\Sigma^{*+} & \frac{\Sigma^{* 0}}{\sqrt{2}} & \Xi^{* 0} \\
\frac{\Sigma^{* 0}}{\sqrt{2}} & \Sigma^{*-} & \Xi^{*-} \\
\Xi^{* 0} & \Xi^{*-} & \sqrt{3} \Omega^{-}
\end{array}\right)\right) .
$$




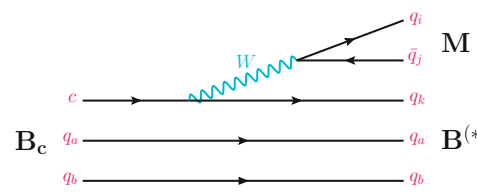

(a) $T$

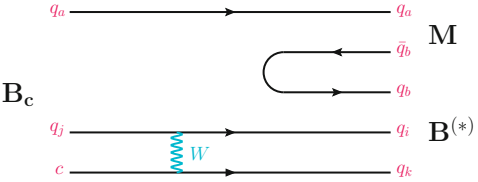

(d) $E^{\prime}$

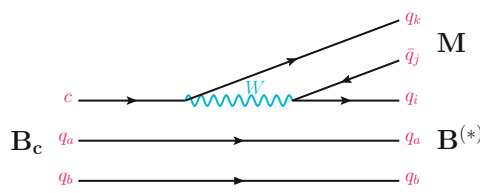

(b) $C$

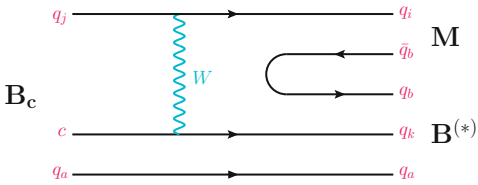

(e) $E_{\mathbf{B}}$

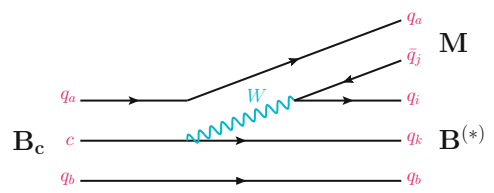

(c) $C^{\prime}$

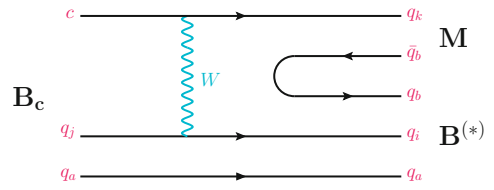

(f) $E_{M}$

Fig. 1 Topological diagrams for the $\mathbf{B}_{c} \rightarrow \mathbf{B}^{(*)} M$ decays

present the full expansions of $T\left(\mathbf{B}_{c} \rightarrow \mathbf{B}^{*} M\right)$ for the CA, SCS and DCS decay modes, respectively. For the branching fractions, we use the equation for the two-body decays, given by [1]

$$
\begin{aligned}
& \mathcal{B}\left(\mathbf{B}_{c} \rightarrow \mathbf{B}^{*} M\right)=\frac{G_{F}^{2}\left|\vec{p}_{\mathbf{B}^{*}}\right| \tau_{\mathbf{B}_{c}}}{16 \pi m_{\mathbf{B}_{c}}^{2}}\left|T\left(\mathbf{B}_{c} \rightarrow \mathbf{B}^{*} M\right)\right|^{2}, \\
& \left|\vec{p}_{\mathbf{B}^{*}}\right|=\frac{\sqrt{\left(m_{\mathbf{B}_{c}}^{2}-m_{+}^{2}\right)\left(m_{\mathbf{B}_{c}}^{2}-m_{-}^{2}\right)}}{2 m_{\mathbf{B}_{c}}},
\end{aligned}
$$

with $m_{ \pm}=m_{\mathbf{B}^{*}} \pm m_{M}$, where $\tau_{\mathbf{B}_{c}}$ stands for the $\mathbf{B}_{c}$ baryon lifetime.

\section{Numerical analysis and discussions}

In the numerical analysis, we adopt the Cabibbo-KobayashiMaskawa (CKM) matrix elements as [1]

$$
\left(V_{c s}, V_{u d}, V_{u s}, V_{c d}\right)=\left(1-\lambda^{2} / 2,1-\lambda^{2} / 2, \lambda,-\lambda\right),
$$

with $\lambda=s_{c}=0.22453 \pm 0.00044$ in the Wolfenstein parameterization. Besides, the $\mathbf{B}_{c}$ and $\mathbf{B}^{*}$ masses, together with the lifetime for $\mathbf{B}_{c}$, are adopted from the PDG [1]. We perform a minimum $\chi^{2}$-fit with $\chi^{2}=\sum\left(\mathcal{B}_{t h}-\mathcal{B}_{e x}\right)^{2} / \sigma_{e x}^{2}$, where $\mathcal{B}_{t h(e x)}$ represents the theoretical (experimental) input of the branching ratio, and $\sigma_{e x}$ the experimental error. We calculate $\mathcal{B}_{t h}$ with the equation in Eq. (9), together with $\left(\mathcal{B}_{e x}, \sigma_{e x}\right)$ from Table 1 . Note that $\mathcal{B}\left(\Xi_{c}^{+} \rightarrow \Sigma^{*+} \bar{K}^{0}, \Xi^{* 0} \pi^{+}\right)$are not involved in the fit.

We use two scenarios for the global fit. In the first scenario $(S 1)$, we take $E_{\mathbf{B}(M)}^{S}=E_{\mathbf{B}(M)}$ under the exact $S U(3)_{f}$ symmetry. Since $E_{\mathbf{B}}$ and $E_{M}$ are complex numbers, it leads to three independent parameters, given by

$\left|E_{\mathbf{B}}\right|,\left|E_{M}\right| e^{i \delta_{E_{M}}}$,

where $E_{\mathbf{B}}$ is set to be real, and $\delta_{E_{M}}$ is a relative strong phase. Using the $\chi^{2}$-fit, we extract that

$$
\left(\left|E_{\mathbf{B}}\right|,\left|E_{M}\right|\right)=(0.41 \pm 0.03,0.34 \pm 0.03) \mathrm{GeV}^{3},
$$

$$
\delta_{E_{M}}=(180.0 \pm 35.8)^{\circ}
$$

with $\chi^{2} / n . d . f=4.5$, where $n . d . f=1$ is the number of the degrees of freedom. For $\delta_{E_{M}}$, its information is from $\mathcal{B}\left(\Lambda_{c}^{+} \rightarrow \Sigma^{*+} \eta\right)$. Although $\delta_{E_{M}}=180^{\circ}$ has induced the largest positive interference between $E_{\mathbf{B}}$ and $E_{M}$, our result of $\mathcal{B}\left(\Lambda_{c}^{+} \rightarrow \Sigma^{*+} \eta\right)=(5.3 \pm 0.7) \times 10^{-3}$ is still shown to be in tension with the observation of $(9.1 \pm 2.0) \times 10^{-3}$. Sizeably, it adds 3.6 to the total $\chi^{2}$ value.

Since $\Lambda_{c}^{+} \rightarrow \Sigma^{*+} \eta$ is in association with $\left|E_{M}^{s}\right|$, the tension hints the broken $S U(3)_{f}$ symmetry, where $\left|E_{M}^{s}\right|$ is not equal to $\left|E_{M}\right|$. On the other hand, $\mathcal{B}\left(\Xi_{c}^{0} \rightarrow \Omega^{-} K^{+}\right)$is fitted to agree with the data, indicating that $\left|E_{\mathbf{B}}^{s}\right|$ is not deviating from $\left|E_{\mathbf{B}}\right|$. Currently, the data points are not sufficient for an independent extraction of $\left|E_{M}^{s}\right|$. We hence adopt the numerical results from the two-body $D$ meson decays, where the similar $W_{\mathrm{EX}}$ contributions have been found to induce the severe $S U(3)_{f}$ symmetry breaking [18-21]. In the second scenario (S2), we take $\left|E_{M}^{S}\right|=n_{q} \times\left|E_{M}\right|$ and $\left|E_{\mathbf{B}}^{S}\right| \simeq\left|E_{\mathbf{B}}\right|$, with $n_{q}=1.4$ adopted from [21]. Consequently, we obtain

$$
\begin{aligned}
& \left(\left|E_{\mathbf{B}}\right|,\left|E_{M}\right|\right)=(0.40 \pm 0.03,0.34 \pm 0.03) \mathrm{GeV}^{3}, \\
& \delta_{E_{M}}=(180.0 \pm 46.8)^{\circ},
\end{aligned}
$$

where $\chi^{2} / n . d . f$ is reduced as 1.3 . As the demonstration, we obtain $\mathcal{B}\left(\Lambda_{c}^{+} \rightarrow \Sigma^{*+} \eta\right)=(7.3 \pm 1.5) \times 10^{-3}$, which alleviates the deviation from the observation. With the fit values of $\left(\left|E_{\mathbf{B}}\right|,\left|E_{M}\right|, \delta_{E_{M}}\right)$ in $S 1$ and $S 2$, we present the branching ratios of the $\mathbf{B}_{c} \rightarrow \mathbf{B}^{*} M$ decays in Tables 1, 2 and 3 , along with the recent theoretical results for comparison.

We get some useful relations in the quark-diagram scheme. For example, we find out three triangle sum rules for $\mathbf{B}_{c} \rightarrow \Delta \pi$, given by

$$
\begin{aligned}
& T\left(\Lambda_{c}^{+} \rightarrow \Delta^{0} \pi^{+}\right)-T\left(\Lambda_{c}^{+} \rightarrow \Delta^{++} \pi^{-}\right) \\
& \quad-\sqrt{6} T\left(\Lambda_{c}^{+} \rightarrow \Delta^{+} \pi^{0}\right)=0, \\
& T\left(\Xi_{c}^{+} \rightarrow \Delta^{0} \pi^{+}\right)-T\left(\Xi_{c}^{+} \rightarrow \Delta^{++} \pi^{-}\right) \\
& \quad-\sqrt{6} T\left(\Xi_{c}^{+} \rightarrow \Delta^{+} \pi^{0}\right)=0,
\end{aligned}
$$


Table 1 Cabibbo-allowed $\mathbf{B}_{c} \rightarrow \mathbf{B}^{*} M$ decays

\begin{tabular}{|c|c|c|c|c|}
\hline Decay modes & $T$-amp & $10^{3} \mathcal{B}(S 1, S 2)$ [our work] & $10^{3} \mathcal{B}\left(S_{p m}, S_{e m}\right)[7]$ & $10^{3} \mathcal{B}_{\text {ex }}[1,35]$ \\
\hline$\Lambda_{c}^{+} \rightarrow \Delta^{++} K^{-}$ & $-\lambda_{a} E_{M}$ & $(12.0 \pm 2.2,11.7 \pm 2.3)$ & $(15.3 \pm 2.4,12.4 \pm 1.0)$ & $10.8 \pm 2.5$ \\
\hline$\Lambda_{c}^{+} \rightarrow \Delta^{+} \bar{K}^{0}$ & $-\lambda_{a} \frac{1}{\sqrt{3}} E_{M}$ & $(4.0 \pm 0.7,3.9 \pm 0.8)$ & $(5.1 \pm 0.8,4.1 \pm 0.3)$ & - \\
\hline$\Lambda_{c}^{+} \rightarrow \Sigma^{* 0} \pi^{+}$ & $-\lambda_{a} \frac{1}{\sqrt{6}} E_{\mathbf{B}}$ & $(2.9 \pm 0.4,2.8 \pm 0.4)$ & $(2.2 \pm 0.4,2.1 \pm 0.2)$ & - \\
\hline$\Lambda_{c}^{+} \rightarrow \Sigma^{*+} \pi^{0}$ & $-\lambda_{a} \frac{1}{\sqrt{6}} E_{\mathbf{B}}$ & $(2.9 \pm 0.4,2.8 \pm 0.4)$ & $(2.2 \pm 0.4,2.1 \pm 0.2)$ & - \\
\hline$\Lambda_{c}^{+} \rightarrow \Sigma^{*+} \eta$ & $-\lambda_{a} \frac{1}{\sqrt{6}}\left(E_{\mathbf{B}} c \phi-\sqrt{2} E_{M}^{(s)} s \phi\right)$ & $(5.3 \pm 0.8,7.3 \pm 1.5)$ & $(3.1 \pm 0.6,6.2 \pm 0.5)$ & $9.1 \pm 2.0$ \\
\hline$\Lambda_{c}^{+} \rightarrow \Sigma^{*+} \eta^{\prime}$ & $-\lambda_{a} \frac{1}{\sqrt{6}}\left(E_{\mathbf{B}} s \phi+\sqrt{2} E_{M}^{(s)} c \phi\right)$ & $(0,0)$ & - & - \\
\hline$\Lambda_{c}^{+} \rightarrow \Xi^{* 0} K^{+}$ & $-\lambda_{a} \frac{1}{\sqrt{3}} E_{\mathbf{B}}$ & $(3.9 \pm 0.6,3.9 \pm 0.6)$ & $(1.0 \pm 0.2,4.1 \pm 0.3)$ & $4.3 \pm 0.9$ \\
\hline$\Xi_{c}^{0} \rightarrow \Sigma^{*+} K^{-}$ & $\lambda_{a} \frac{1}{\sqrt{3}} E_{M}$ & $(1.8 \pm 0.3,1.7 \pm 0.3)$ & $(3.1 \pm 0.5,2.3 \pm 0.2)$ & - \\
\hline$\Xi_{c}^{0} \rightarrow \Sigma^{* 0} \bar{K}^{0}$ & $\lambda_{a} \frac{1}{\sqrt{6}} E_{M}$ & $(0.9 \pm 0.2,0.9 \pm 0.2)$ & $(1.6 \pm 0.2,1.2 \pm 0.1)$ & - \\
\hline$\Xi_{c}^{0} \rightarrow \Xi^{*-} \pi^{+}$ & $\lambda_{a} \frac{1}{\sqrt{3}} E_{\mathbf{B}}$ & $(2.6 \pm 0.4,2.5 \pm 0.4)$ & $(2.8 \pm 0.5,2.3 \pm 0.2)$ & - \\
\hline$\Xi_{c}^{0} \rightarrow \Xi^{* 0} \pi^{0}$ & $\lambda_{a} \frac{1}{\sqrt{6}} E_{\mathbf{B}}$ & $(1.3 \pm 0.2,1.3 \pm 0.2)$ & $(1.4 \pm 0.2,1.2 \pm 0.1)$ & - \\
\hline$\Xi_{c}^{0} \rightarrow \Xi^{* 0} \eta$ & $\lambda_{a} \frac{1}{\sqrt{6}}\left(E_{\mathbf{B}} c \phi-\sqrt{2} E_{M}^{(s)} s \phi\right)$ & $(2.4 \pm 0.4,3.4 \pm 0.7)$ & $(2.1 \pm 0.4,3.5 \pm 0.3)$ & - \\
\hline$\Xi_{c}^{0} \rightarrow \Xi^{* 0} \eta^{\prime}$ & $\lambda_{a} \frac{1}{\sqrt{6}}\left(E_{\mathbf{B}} s \phi+\sqrt{2} E_{M}^{(s)} c \phi\right)$ & $(0.01 \pm 0.04,0.08 \pm 0.10)$ & - & - \\
\hline$\Xi_{c}^{0} \rightarrow \Omega^{-} K^{+}$ & $\lambda_{a} E_{\mathbf{B}}^{(s)}$ & $(4.8 \pm 0.7,4.8 \pm 0.7)$ & $(2.3 \pm 0.5,7.0 \pm 0.6)$ & $4.2 \pm 1.0$ \\
\hline$\Xi_{c}^{+} \rightarrow \Sigma^{*+} \bar{K}^{0}$ & 0 & 0 & 0 & $28.6 \pm 16.8$ \\
\hline$\Xi_{c}^{+} \rightarrow \Xi^{* 0} \pi^{+}$ & 0 & 0 & 0 & $<4.0$ \\
\hline
\end{tabular}

$$
\begin{aligned}
& T\left(\Xi_{c}^{0} \rightarrow \Delta^{+} \pi^{-}\right)-T\left(\Xi_{c}^{0} \rightarrow \Delta^{-} \pi^{+}\right) \\
& \quad-\sqrt{6} T\left(\Xi_{c}^{0} \rightarrow \Delta^{0} \pi^{0}\right)=0 .
\end{aligned}
$$

Besides, we obtain

$$
\begin{aligned}
& T\left(\Lambda_{c}^{+} \rightarrow \Delta^{+} K^{0}, \Delta^{0} K^{+}\right)=0, \\
& T\left(\Xi_{c}^{+} \rightarrow \Sigma^{*+} \bar{K}^{0}, \Xi^{* 0} \pi^{+}\right)=0,
\end{aligned}
$$

as the consequence of $C^{\prime}$ being set to give no contribution to $\mathbf{B}_{c} \rightarrow \mathbf{B}^{*} M$. Indeed, $C^{\prime}$ is the only topology that takes part in the decays in Eq. (15), but suppressed due to the Körner-PatiWoo theorem [32-34]. According to the other theoretical calculations [6,7,22-24], $\mathcal{B}\left(\Xi_{c}^{+} \rightarrow \Sigma^{*+} \bar{K}^{0}, \Xi^{* 0} \pi^{+}\right)=0$ is also predicted, which supports that $C^{\prime}=0$. Experimentally, $\mathcal{B}_{\text {ex }}\left(\Xi_{c}^{+} \rightarrow \Sigma^{*+} \bar{K}^{0}, \Xi^{* 0} \pi^{+}\right)$in Table 2 can be used to test the suppression. With $\mathcal{B}\left(\Xi_{c}^{+} \rightarrow \Xi^{* 0} \pi^{+}\right) / \mathcal{B}\left(\Xi_{c}^{+} \rightarrow\right.$ $\left.\Xi^{-} \pi^{+} \pi^{+}\right)<0.1$ and $\mathcal{B}\left(\Xi_{c}^{+} \rightarrow \Xi^{-} \pi^{+} \pi^{+}\right)=(2.86 \pm$ $1.21 \pm 0.38) \times 10^{-2}[1,35]$, we determine $\mathcal{B}_{\text {ex }}\left(\Xi_{c}^{+} \rightarrow\right.$ $\left.\Xi^{* 0} \pi^{+}\right)<4.0 \times 10^{-3}$, which can be seen as the nonobservation to agree with $T\left(\Xi_{c}^{+} \rightarrow \Xi^{* 0} \pi^{+}\right)=0$. However, $\mathcal{B}_{\text {ex }}\left(\Xi_{c}^{+} \rightarrow \Sigma^{*+} \bar{K}^{0}\right)=(2.9 \pm 1.7) \times 10^{-2}$ seems to disagree with the prediction of $\mathcal{B}\left(\Xi_{c}^{+} \rightarrow \Sigma^{*+} \bar{K}^{0}\right)=0$, despite of the large uncertainty. While the Körner-Pati-Woo theorem is regarded as a tree-level approximation, allowing possible corrections to $C^{\prime}$ and $E^{\prime}$, we need more accurate observations to test if $C^{\prime}\left(E^{\prime}\right)=0$.

Uniquely, the decuplet baryon can contain three identical quarks, denoted by $\mathbf{B}^{*}(q q q)$, which leads to an additional weight factor of $\sqrt{3}$ among the decuplet baryons in Eq. (6).
The factor can be considered as the main reason why $\Lambda_{c}^{+} \rightarrow$ $\Delta^{++} K^{-}$and $\Xi_{c}^{0} \rightarrow \Omega^{-} K^{+}$are measured with the largest branching fractions in the CA decay channels of $\Lambda_{c}^{+}, \Xi_{c}^{0} \rightarrow$ $\mathbf{B}^{*} M$, respectively. Accordingly, the $T$-amps with $\mathbf{B}^{*}(q q q)$ are listed as

$$
\begin{aligned}
& T\left(\Lambda_{c}^{+} \rightarrow \Delta^{++} K^{-}, \Delta^{++} \pi^{-}\right)=-\left(\lambda_{a}, \lambda_{d}\right) E_{M}, \\
& T\left(\Xi_{c}^{+} \rightarrow \Delta^{++} K^{-}, \Delta^{++} \pi^{-}\right)=-\left(\lambda_{s}, \lambda_{c}\right) E_{M}, \\
& T\left(\Xi_{c}^{0} \rightarrow \Omega^{-} K^{+}, \Delta^{-} \pi^{+}\right)=\left(\lambda_{a},-\lambda_{c}\right) E_{\mathbf{B}} .
\end{aligned}
$$

While $\mathcal{B}\left(\Lambda_{c}^{+} \rightarrow \Delta^{++} K^{-}\right)$and $\mathcal{B}\left(\Xi_{c}^{0} \rightarrow \Omega^{-} K^{+}\right)$have been observed, the other branching fractions are given by

$$
\begin{gathered}
\mathcal{B}\left(\Lambda_{c}^{+} \rightarrow \Delta^{++} \pi^{-}, \Xi_{c}^{+} \rightarrow \Delta^{++} K^{-}\right) \\
\quad=(7.0 \pm 1.4,13.5 \pm 2.7) \times 10^{-4} \\
\mathcal{B}\left(\Xi_{c}^{+} \rightarrow \Delta^{++} \pi^{-}, \Xi_{c}^{0} \rightarrow \Delta^{-} \pi^{+}\right) \\
\quad=(7.8 \pm 1.6,2.5 \pm 0.4) \times 10^{-5}
\end{gathered}
$$

which are predicted as the largest branching fractions in the $\operatorname{SCS} \Lambda_{c}^{+}\left(\Xi_{c}^{+}\right)$and DCS $\Xi_{c}^{+(0)}$ decay channels, respectively. Here, we present our predictions of $S 2$, which is favored by the $\chi^{2}$-fit. The equality of $T\left(\Lambda_{c}^{+} \rightarrow \Sigma^{* 0} \pi^{+}\right)=T\left(\Lambda_{c}^{+} \rightarrow\right.$ $\Sigma^{*+} \pi^{0}$ ) corresponds to the isospin symmetry. The branching fraction, given by

$\mathcal{B}\left(\Lambda_{c}^{+} \rightarrow \Sigma^{* 0(+)} \pi^{+(0)}\right)=(2.8 \pm 0.4) \times 10^{-3}$,

can be used to test the broken effect. The decays $\mathbf{B}_{c} \rightarrow$ $\mathbf{B}^{*} \eta^{(\prime)}, \Lambda_{c}^{+} \rightarrow \Sigma^{*+} \eta, \Xi_{c}^{0} \rightarrow \Xi^{* 0} \eta$ and $\Xi_{c}^{+} \rightarrow \Sigma^{*+} \eta$ 
Table 2 Singly Cabibbo-suppressed $\mathbf{B}_{c} \rightarrow \mathbf{B}^{*} M$ decays

\begin{tabular}{|c|c|c|c|}
\hline Decay modes & $T$-amp & $10^{4} \mathcal{B}(S 1, S 2)$ [our work] & $10^{4} \mathcal{B}\left(S_{p m}, S_{e m}\right)[7]$ \\
\hline$\Lambda_{c}^{+} \rightarrow \Delta^{++} \pi^{-}$ & $-\lambda_{d} E_{M}$ & $(7.2 \pm 1.3,7.0 \pm 1.4)$ & $(12.5 \pm 2.0,6.6 \pm 0.6)$ \\
\hline$\Lambda_{c}^{+} \rightarrow \Delta^{+} \pi^{0}$ & $-\lambda_{d} \frac{1}{\sqrt{6}}\left(E_{\mathbf{B}}-E_{M}\right)$ & $(5.8 \pm 0.9,5.6 \pm 1.1)$ & $(8.3 \pm 1.3,4.4 \pm 0.4)$ \\
\hline$\Lambda_{c}^{+} \rightarrow \Delta^{0} \pi^{+}$ & $-\lambda_{d} \frac{1}{\sqrt{3}} E_{\mathbf{B}}$ & $(3.5 \pm 0.5,3.3 \pm 0.5)$ & $(4.2 \pm 0.7,2.2 \pm 0.2)$ \\
\hline$\Lambda_{c}^{+} \rightarrow \Delta^{+} \eta$ & $-\lambda_{d} \frac{1}{\sqrt{6}}\left(E_{\mathbf{B}}+E_{M}\right) c \phi$ & $(0.03 \pm 0.28,0.02 \pm 0.45)$ & - \\
\hline$\Lambda_{c}^{+} \rightarrow \Delta^{+} \eta^{\prime}$ & $-\lambda_{d} \frac{1}{\sqrt{6}}\left(E_{\mathbf{B}}+E_{M}\right) s \phi$ & $(0.01 \pm 0.09,0.01 \pm 0.15)$ & - \\
\hline$\Lambda_{c}^{+} \rightarrow \Sigma^{*+} K^{0}$ & $-\lambda_{d} \frac{1}{\sqrt{3}} E_{M}^{(s)}$ & $(1.8 \pm 0.4,3.5 \pm 0.7)$ & $(1.3 \pm 0.2,2.2 \pm 0.2)$ \\
\hline$\Lambda_{c}^{+} \rightarrow \Sigma^{* 0} K^{+}$ & $-\lambda_{d} \frac{1}{\sqrt{6}} E_{\mathbf{B}}^{(s)}$ & $(1.3 \pm 0.2,1.3 \pm 0.2)$ & $(0.7 \pm 0.1,1.1 \pm 0.1)$ \\
\hline$\Xi_{c}^{0} \rightarrow \Delta^{+} K^{-}$ & $-\lambda_{s} \frac{1}{\sqrt{3}} E_{M}$ & $(1.1 \pm 0.2,1.0 \pm 0.2)$ & $(3.0 \pm 0.5,1.2 \pm 0.1)$ \\
\hline$\Xi_{c}^{0} \rightarrow \Delta^{0} \bar{K}^{0}$ & $-\lambda_{s} \frac{1}{\sqrt{3}} E_{M}$ & $(1.1 \pm 0.2,1.0 \pm 0.2)$ & $(3.0 \pm 0.5,1.2 \pm 0.1)$ \\
\hline$\Xi_{c}^{0} \rightarrow \Sigma^{*-} \pi^{+}$ & $\frac{1}{\sqrt{3}}\left(\lambda_{d} E_{\mathbf{B}}-\lambda_{s} E_{\mathbf{B}}\right)$ & $(6.1 \pm 0.9,5.8 \pm 0.9)$ & $(9.9 \pm 1.6,4.9 \pm 0.4)$ \\
\hline$\Xi_{c}^{0} \rightarrow \Sigma^{*+} \pi^{-}$ & $\lambda_{d} \frac{1}{\sqrt{3}} E_{M}$ & $(1.0 \pm 0.2,1.0 \pm 0.2)$ & $(2.5 \pm 0.4,1.2 \pm 0.1)$ \\
\hline$\Xi_{c}^{0} \rightarrow \Sigma^{* 0} \pi^{0}$ & $\frac{1}{\sqrt{12}}\left[\lambda_{d}\left(E_{\mathbf{B}}-E_{M}\right)-\lambda_{s} E_{\mathbf{B}}\right]$ & $(3.1 \pm 0.4,2.9 \pm 0.5)$ & $(5.6 \pm 0.9,2.8 \pm 0.2)$ \\
\hline$\Xi_{c}^{0} \rightarrow \Sigma^{* 0} \eta$ & $\frac{1}{\sqrt{12}}\left[\lambda_{d}\left(E_{\mathbf{B}}+E_{M}\right) c \phi+\lambda_{s}\left(\sqrt{2} E_{M}^{(s)} s \phi-E_{\mathbf{B}} c \phi\right)\right]$ & $(0.9 \pm 0.2,1.2 \pm 0.3)$ & $(1.1 \pm 0.2,0.9 \pm 0.1)$ \\
\hline$\Xi_{c}^{0} \rightarrow \Sigma^{* 0} \eta^{\prime}$ & $\frac{1}{\sqrt{12}}\left[\lambda_{d}\left(E_{\mathbf{B}}+E_{M}\right) s \phi-\lambda_{s}\left(\sqrt{2} E_{M}^{(s)} c \phi+E_{\mathbf{B}} s \phi\right)\right]$ & $(0.004 \pm 0.120,0.050 \pm 0.250)$ & - \\
\hline$\Xi_{c}^{0} \rightarrow \Xi^{* 0} K^{0}$ & $\frac{1}{\sqrt{3}} \lambda_{d} E_{M}^{(s)}$ & $(0.8 \pm 0.2,1.6 \pm 0.4)$ & $(0.9 \pm 0.2,1.2 \pm 0.1)$ \\
\hline$\Xi_{c}^{0} \rightarrow \Xi^{*-} K^{+}$ & $\frac{1}{\sqrt{3}}\left(\lambda_{d} E_{\mathbf{B}}^{(s)}-\lambda_{s} E_{\mathbf{B}}^{(s)}\right)$ & $(4.6 \pm 0.7,4.6 \pm 0.7)$ & $(3.6 \pm 0.6,4.9 \pm 0.4)$ \\
\hline$\Xi_{c}^{+} \rightarrow \Delta^{++} K^{-}$ & $-\lambda_{s} E_{M}$ & $(13.8 \pm 2.5,13.5 \pm 2.7)$ & $(35.0 \pm 5.7,14.6 \pm 1.2)$ \\
\hline$\Xi_{c}^{+} \rightarrow \Delta^{+} \bar{K}^{0}$ & $-\lambda_{s} \frac{1}{\sqrt{3}} E_{M}$ & $(4.6 \pm 0.8,4.5 \pm 0.9)$ & $(11.7 \pm 1.9,4.9 \pm 0.4)$ \\
\hline$\Xi_{c}^{+} \rightarrow \Sigma^{*+} \pi^{0}$ & $-\lambda_{s} \frac{1}{\sqrt{6}} E_{\mathbf{B}}$ & $(3.4 \pm 0.5,3.2 \pm 0.5)$ & $(4.8 \pm 0.8,2.4 \pm 0.2)$ \\
\hline$\Xi_{c}^{+} \rightarrow \Sigma^{* 0} \pi^{+}$ & $-\lambda_{s} \frac{1}{\sqrt{6}} E_{\mathbf{B}}$ & $(3.4 \pm 0.5,3.2 \pm 0.5)$ & $(4.8 \pm 0.8,2.4 \pm 0.2)$ \\
\hline$\Xi_{c}^{+} \rightarrow \Sigma^{*+} \eta$ & $-\lambda_{s} \frac{1}{\sqrt{6}}\left(E_{\mathbf{B}} c \phi-\sqrt{2} E_{M}^{(s)} s \phi\right)$ & $(6.4 \pm 1.0,9.1 \pm 1.8)$ & $(8.7 \pm 1.4,7.3 \pm 0.6)$ \\
\hline$\Xi_{c}^{+} \rightarrow \Sigma^{*+} \eta^{\prime}$ & $-\lambda_{s} \frac{1}{\sqrt{6}}\left(E_{\mathbf{B}} s \phi+\sqrt{2} E_{M}^{(s)} c \phi\right)$ & $(0.1 \pm 0.3,0.6 \pm 0.8)$ & - \\
\hline$\Xi_{c}^{+} \rightarrow \Xi^{* 0} K^{+}$ & $-\lambda_{s} \frac{1}{\sqrt{3}} E_{\mathbf{B}}^{(s)}$ & $(5.0 \pm 0.8,5.0 \pm 0.8)$ & $(3.5 \pm 0.6,4.9 \pm 0.4)$ \\
\hline
\end{tabular}

have sizeable branching fractions, which is due to the constructive interferences between $E_{\mathbf{B}}$ and $E_{M}$. However, the other branching fractions of $\mathbf{B}_{c} \rightarrow \mathbf{B}^{*} \eta^{(\prime)}$ are typically small with the destructive interferences. Moreover, we find that $\mathcal{B}\left(\Lambda_{c}^{+} \rightarrow \Sigma^{*+} \eta^{\prime}\right)=0$ with $m_{\Lambda_{c}^{+}}<m_{\Sigma^{*+}}+m_{\eta^{\prime}}$.

The approach of the irreducible $S U(3)_{f}$ symmetry has been widely used in the hadron weak decays [4-16]. For $\mathbf{B}_{c} \rightarrow \mathbf{B}^{*} M$, there exist four parameters $a_{8}$ and $a_{9,10,11}[4,6]$, which correspond to the decomposition of $\mathcal{H}_{\text {eff }}=H(6)+$ $H(\overline{15})$ in the $S U(3)_{f}$ representation of 6 and $\overline{15}$, respectively. By comparison, we derive that

$$
\begin{aligned}
\left(E_{\mathbf{B}}, E_{M}\right) & =\left(-2 a_{8}+a_{9}, 2 a_{8}+a_{9}\right),\left(E^{\prime}, C^{\prime}\right) \\
& =\left(-2 a_{9}-2 a_{10},-2 a_{11}\right),
\end{aligned}
$$

such that $a_{i}$ are found to correspond to the topologies. Since $\left(E^{\prime}, C^{\prime}\right)$ have been the vanishing topological parameters, one has $a_{9}=-a_{10}$ and $a_{11}=0$. Moreover, our global fits for
$E_{\mathbf{B}, M}$ indicate that $a_{9(10)}$ from $H(\overline{15})$ has a non-zero value. By contrast, the numerical analysis performed with the irreducible $S U(3)_{f}$ symmetry neglects the contributions from $H(\overline{15})$ [7], whose results are given in the tables. In the physical mass scenario $\left(S_{p m}\right)$ for the global fit in Ref. [7], where $m_{\mathbf{B}_{c}}, m_{\mathbf{B}^{*}}$ and $m_{M}$ are taken from the physical values in Ref. [1], $\mathcal{B}\left(\Lambda_{c}^{+} \rightarrow \Sigma^{*+} \eta, \Xi^{* 0} K^{+}\right)$and $\mathcal{B}\left(\Xi_{c}^{0} \rightarrow \Omega^{-} K^{+}\right)$ are fitted to be a few times smaller than the observations. Instead of considering the $S U(3)_{f}$ symmetry breaking, one performs another fit in the equal mass scenario $\left(S_{e m}\right)$, where $m_{\Lambda_{c}}=m_{\Xi_{c}}, m_{\Delta}=m_{\Sigma^{*}}=m_{\Omega}$ and $m_{\pi}=m_{\eta}=m_{K}$, resulting in the raised values of the above branching fractions.

\section{Conclusions}

In summary, we have studied the $\mathbf{B}_{c} \rightarrow \mathbf{B}^{*} M$ decays in the quark-diagram scheme. We have found that only two $W$ - 
Table 3 Doubly Cabibbo-suppressed $\mathbf{B}_{c} \rightarrow \mathbf{B}^{*} M$ decays

\begin{tabular}{|c|c|c|c|}
\hline Decay modes & $T$-amp & $10^{5} \mathcal{B}(S 1, S 2)$ [our work] & $10^{5} \mathcal{B}\left(S_{p m}, S_{e m}\right)[7]$ \\
\hline$\Lambda_{c}^{+} \rightarrow \Delta^{+} K^{0}$ & 0 & 0 & 0 \\
\hline$\Lambda_{c}^{+} \rightarrow \Delta^{0} K^{+}$ & 0 & 0 & 0 \\
\hline$\Xi_{c}^{0} \rightarrow \Delta^{+} \pi^{-}$ & $-\lambda_{c} \frac{1}{\sqrt{3}} E_{M}$ & $(0.6 \pm 0.1,0.6 \pm 0.1)$ & $(2.2 \pm 0.4,0.7 \pm 0.1)$ \\
\hline$\Xi_{c}^{0} \rightarrow \Delta^{0} \pi^{0}$ & $-\lambda_{c} \frac{1}{\sqrt{6}}\left(E_{\mathbf{B}}-E_{M}\right)$ & $(1.5 \pm 0.2,1.4 \pm 0.3)$ & $(4.3 \pm 0.7,1.3 \pm 0.1)$ \\
\hline$\Xi_{c}^{0} \rightarrow \Delta^{-} \pi^{+}$ & $-\lambda_{c} E_{\mathbf{B}}$ & $(2.7 \pm 0.4,2.5 \pm 0.4)$ & $(6.5 \pm 1.1,2.0 \pm 0.2)$ \\
\hline$\Xi_{c}^{0} \rightarrow \Delta^{0} \eta$ & $-\lambda_{c} \frac{1}{\sqrt{6}}\left(E_{\mathbf{B}}+E_{M}\right) c \phi$ & $(0.01 \pm 0.07,0.01 \pm 0.12)$ & - \\
\hline$\Xi_{c}^{0} \rightarrow \Delta^{0} \eta^{\prime}$ & $-\lambda_{c} \frac{1}{\sqrt{6}}\left(E_{\mathbf{B}}+E_{M}\right) s \phi$ & $(0.003 \pm 0.034,0.003 \pm 0.055)$ & - \\
\hline$\Xi_{c}^{0} \rightarrow \Sigma^{* 0} K^{0}$ & $-\lambda_{c} \frac{1}{\sqrt{6}} E_{M}^{(s)}$ & $(0.2 \pm 0.1,0.5 \pm 0.1)$ & $(0.4 \pm 0.1,0.3 \pm 0.0)$ \\
\hline$\Xi_{c}^{0} \rightarrow \Sigma^{*-} K^{+}$ & $-\lambda_{c} \frac{1}{\sqrt{3}} E_{\mathbf{B}}^{(s)}$ & $(0.7 \pm 0.1,0.7 \pm 0.1)$ & $(0.9 \pm 0.1,0.7 \pm 0.1)$ \\
\hline$\Xi_{c}^{+} \rightarrow \Delta^{++} \pi^{-}$ & $-\lambda_{c} E_{M}$ & $(8.0 \pm 1.5,7.8 \pm 1.6)$ & $(25.5 \pm 4.4,7.8 \pm 0.7)$ \\
\hline$\Xi_{c}^{+} \rightarrow \Delta^{+} \pi^{0}$ & $-\lambda_{c} \frac{1}{\sqrt{6}}\left(E_{\mathbf{B}}-E_{M}\right)$ & $(6.5 \pm 1.0,6.3 \pm 1.2)$ & $(17.0 \pm 2.9,5.2 \pm 0.4)$ \\
\hline$\Xi_{c}^{+} \rightarrow \Delta^{0} \pi^{+}$ & $-\lambda_{c} \frac{1}{\sqrt{3}} E_{\mathbf{B}}$ & $(3.9 \pm 0.6,3.7 \pm 0.7)$ & $(8.5 \pm 1.5,2.6 \pm 0.2)$ \\
\hline$\Xi_{c}^{+} \rightarrow \Delta^{+} \eta$ & $-\lambda_{c} \frac{1}{\sqrt{6}}\left(E_{\mathbf{B}}+E_{M}\right) c \phi$ & $(0.03 \pm 0.32,0.03 \pm 0.52)$ & - \\
\hline$\Xi_{c}^{+} \rightarrow \Delta^{+} \eta^{\prime}$ & $-\lambda_{c} \frac{1}{\sqrt{6}}\left(E_{\mathbf{B}}+E_{M}\right) s \phi$ & $(0.01 \pm 0.15,0.01 \pm 0.24)$ & - \\
\hline$\Xi_{c}^{+} \rightarrow \Sigma^{*+} K^{0}$ & $-\lambda_{c} \frac{1}{\sqrt{3}} E_{M}^{(s)}$ & $(2.1 \pm 0.4,4.2 \pm 0.8)$ & $(3.5 \pm 0.6,2.6 \pm 0.2)$ \\
\hline$\Xi_{c}^{+} \rightarrow \Sigma^{* 0} K^{+}$ & $-\lambda_{c} \frac{1}{\sqrt{6}} E_{\mathbf{B}}^{(s)}$ & $(1.5 \pm 0.2,1.5 \pm 0.2)$ & $(1.7 \pm 0.3,1.3 \pm 0.1)$ \\
\hline
\end{tabular}

exchange diagrams, $E_{\mathbf{B}}$ and $E_{M}$, could give contributions to the observed branching fractions of $\Lambda_{c}^{+} \rightarrow\left(\Delta^{++} K^{-}\right.$, $\left.\Sigma^{*+} \eta, \Xi^{* 0} K^{+}\right)$and $\Xi_{c}^{0} \rightarrow \Omega^{-} K^{+}$. In addition, we have predicted $\mathcal{B}\left(\Lambda_{c}^{+} \rightarrow \Sigma^{* 0(+)} \pi^{+(0)}\right)=(2.8 \pm 0.4) \times 10^{-3}$, which respects the isospin symmetry. To interpret the observation of $\mathcal{B}\left(\Lambda_{c}^{+} \rightarrow \Sigma^{*+} \eta\right)$, we have taken into account the $S U(3)_{f}$ symmetry breaking. In particular, $\mathcal{B}\left(\Lambda_{c}^{+} \rightarrow\right.$ $\left.\Delta^{++} \pi^{-}, \Xi_{c}^{+} \rightarrow \Delta^{++} K^{-}\right)=(7.0 \pm 1.4,13.5 \pm 2.7) \times 10^{-4}$ and $\mathcal{B}\left(\Xi_{c}^{+} \rightarrow \Delta^{++} \pi^{-}, \Xi_{c}^{0} \rightarrow \Delta^{-} \pi^{+}\right)=(7.8 \pm 1.6,2.5 \pm$ $0.4) \times 10^{-5}$ have been predicted as the largest branching fractions in the SCS $\Lambda_{c}^{+}\left(\Xi_{c}^{+}\right)$and DSC $\Xi_{c}^{+(0)}$ decay channels, respectively.

Acknowledgements We would like to thank Prof. X.G. He for useful discussions. This work was supported by National Science Foundation of China (11675030).

Data Availability Statement This manuscript has no associated data or the data will not be deposited. [Authors' comment: The data used in this paper are publicly available and they can be found in the corresponding references.]

Open Access This article is licensed under a Creative Commons Attribution 4.0 International License, which permits use, sharing, adaptation, distribution and reproduction in any medium or format, as long as you give appropriate credit to the original author(s) and the source, provide a link to the Creative Commons licence, and indicate if changes were made. The images or other third party material in this article are included in the article's Creative Commons licence, unless indicated otherwise in a credit line to the material. If material is not included in the article's Creative Commons licence and your intended use is not permitted by statutory regulation or exceeds the permit- ted use, you will need to obtain permission directly from the copyright holder. To view a copy of this licence, visit http://creativecomm ons.org/licenses/by/4.0/.

Funded by $\mathrm{SCOAP}^{3}$.

\section{References}

1. M. Tanabashi et al., [Particle Data Group]. Phys. Rev. D 98, 030001 (2018)

2. A. Zupanc et al., [Belle Collaboration]. Phys. Rev. Lett. 113, 042002 (2014)

3. M. Ablikim et al., [BESIII Collaboration]. Phys. Rev. Lett. 116, 052001 (2016)

4. M.J. Savage, R.P. Springer, Phys. Rev. D 42, 1527 (1990)

5. M.J. Savage, Phys. Lett. B 257, 414 (1991)

6. C.Q. Geng, Y.K. Hsiao, C.W. Liu, T.H. Tsai, JHEP 1711, 147 (2017)

7. C.Q. Geng, C.W. Liu, T.H. Tsai, Y. Yu, Phys. Rev. D 99, 114022 (2019)

8. X.G. He, Y.K. Hsiao, J.Q. Shi, Y.L. Wu, Y.F. Zhou, Phys. Rev. D 64, $034002(2001)$

9. H.K. Fu, X.G. He, Y.K. Hsiao, Phys. Rev. D 69, 074002 (2004)

10. Y.K. Hsiao, C.F. Chang, X.G. He, Phys. Rev. D 93, 114002 (2016)

11. J. Pan, Y.K. Hsiao, J. Sun, X.G. He, Phys. Rev. D 102, 056005 (2020)

12. C.D. Lu, W. Wang, F.S. Yu, Phys. Rev. D 93, 056008 (2016)

13. C.Q. Geng, Y.K. Hsiao, Y.H. Lin, L.L. Liu, Phys. Lett. B 776, 265 (2017)

14. C.Q. Geng, Y.K. Hsiao, C.W. Liu, T.H. Tsai, Phys. Rev. D 97, 073006 (2018)

15. C.Q. Geng, Y.K. Hsiao, C.W. Liu, T.H. Tsai, Phys. Rev. D 99, 073003 (2019) 
16. Y.K. Hsiao, Y. Yu, H.J. Zhao, Phys. Lett. B 792, 35 (2019)

17. H.J. Zhao, Y.L. Wang, Y.K. Hsiao, Y. Yu, JHEP 2002, 165 (2020)

18. H.Y. Cheng, C.W. Chiang, Phys. Rev. D 86, 014014 (2012)

19. Hn Li, C.D. Lu, F.S. Yu, Phys. Rev. D 86, 036012 (2012)

20. Hn Li, C.D. Lu, Q. Qin, F.S. Yu, Phys. Rev. D 89, 054006 (2014)

21. H.Y. Cheng, C.W. Chiang, Phys. Rev. D 100, 093002 (2019)

22. Q.P. Xu, A.N. Kamal, Phys. Rev. D 46, 3836 (1992)

23. J.G. Korner, M. Kramer, Z. Phys. C 55, 659 (1992)

24. K.K. Sharma, R.C. Verma, Phys. Rev. D 55, 7067 (1997)

25. Y. Kohara, Phys. Rev. D 44, 2799 (1991)

26. L.L. Chau, H.Y. Cheng, B. Tseng, Phys. Rev. D 54, 2132 (1996)

27. X.G. He, Y.J. Shi, W. Wang, Eur. Phys. J. C 80, 359 (2020)

28. A.J. Buras, arXiv:hep-ph/9806471

29. T. Feldmann, P. Kroll, B. Stech, Phys. Rev. D 58, 114006 (1998)
30. T. Feldmann, P. Kroll, B. Stech, Phys. Lett. B 449, 339 (1999)

31. Y.K. Hsiao, L. Yang, C.C. Lih, S.Y. Tsai, arXiv:2009.12752 [hep$\mathrm{ph}]$

32. K. Miura, T. Minamikawa, Prog. Theor. Phys. 38, 954 (1967)

33. J.G. Korner, Nucl. Phys. B 25, 282 (1971)

34. J.C. Pati, C.H. Woo, Phys. Rev. D 3, 2920 (1971)

35. Y.B. Li et al., [Belle Collaboration]. Phys. Rev. D 100, 031101 (2019) 